\title{
Evaluación de un sistema de filtros de cascarilla de arroz y luffa cylindrica para el tratamiento de aguas lluvias
}

\author{
Andrés Suárez, ${ }^{1 *}$ Paola Mesa, ${ }^{2}$ Víctor Bravo ${ }^{3}$ y Andrés Prieto ${ }^{4}$
}

\author{
'Universidad Jorge Tadeo Lozano, Facultad de Ciencias Naturales e Ingeniería, Departamento de Ingeniería. Cra. 4 № 22-61 Bogotá D. C., Colombia \\ *Autor para correspondencia: andresf.suareze@utadeo.edu.co \\ Universidad Juan N. Corpas. Facultad de Medicina \\ ${ }^{3}$ Cormacarena. Grupo Aire y Urbano \\ ${ }^{4}$ Interventor relleno Doña Juana
}

\section{Resumen}

Se evaluó un sistema de tratamiento para agua lluvia cosechada en los techos de la Universidad Libre, sede Bosque Popular empleando elementos lignocelulósicos mediante dos filtros de flujo descendente con lecho profundo. Uno que consta de luffa cylindrica como soporte combinado con cascarilla de arroz como medio filtrante y otro que solo funcionó con cascarilla de arroz como medio filtrante.

La eficiencia del tratamiento se evaluó a través de análisis físicos, químicos y biológicos como pH, turbiedad, sólidos disueltos totales, sólidos suspendidos totales, DQO y coliformes para un período de 10 días. Algunos de estos parámetros no mejoraron con el sistema en el período de prueba. Se observó además la ausencia de alcalinidad y dureza, lo que puede permitir el uso de esta agua en procesos diferentes al de consumo humano.

Palabras clave: sistema de filtración, luffa cilíndrica, cascarilla de arroz, agua lluvia.

Editora: Pataquiva-Mateus, A. Y.

Citation: Suárez, A., Mesa, P., Bravo, V. y Prieto, A. (2015). Evaluación de un humedal artificial de flujo subsuperficial para el tratamiento de aguas residuales domésticas. Revista Mutis 5(1); pag 21-27.

Received: May 15, 2015; Accepted: June 5, 2015; Published on line: June 30,2015

Copyright: $\odot 2015$ Suárez et al. This is an open-access article, which permits unrestricted use, distributions and reproduction in any medium, provided the original author and source are credited.

Competing Interests: The authors have no conflict of interest.

\section{Evaluation of filtration system of rice husk and luffa cylindrica for the treatment of harvested rainwater}

\begin{abstract}
Filtration treatment of harvested rainwater with lignocellulose material was evaluated in order to determinate the usage of this water source in the Libre University. Two descending flow filter were used, one with luffa cylindrica as support media combined with rice husk as filtrating media and the other one with rice husk and no support.

The treatment efficiency was established with physical, chemical and biological analysis of the water as $\mathrm{pH}$, turbidity, solids dissolved and suspended, COD, total and fecal coliforms. The variation of those parameters
\end{abstract}


were evaluated in a period of 10 days. Some of those parameters did not show an improvement with the system. The absence of hardness and acidity in this water might allow the usage of this water for process besides the human consumption.

Keywords: filtration system, luffa cilíndrica, rice husk, rain water.

\section{Introducción}

La carencia de agua en el mundo ha llevado a buscar alternativas para la captación de este vital líquido, además de propender por la conservación del mismo. Por tal motivo se hace necesario incursionar en procesos de cosecha del agua lluvia, que implica procesos de captación, almacenamiento y conservación. Anteriormente se usaban simples técnicas de almacenamiento como jarras y ollas. Esta tecnología "antigua" está ganando popularidad en estos días de climas extremos y en lugares apartados sin infraestructuras adecuadas para la producción de agua potable especialmente en las zonas de períodos prolongados de lluvia escasa o nula (Guozhen, Yuanchao, Xiaodong \& Weina, 2011).

Según evaluaciones del IDEAM, cerca del $50 \%$ de la población colombiana que vive en las áreas urbanas municipales está expuesta a sufrir problemas de suministro de agua, como consecuencia de la presión sobre las cuencas hidrográficas y las restricciones de uso por contaminación de las aguas superficiales (Ballén, Galarza, \& Ortiz, 2006). El agua de lluvia recolectada es una valiosa fuente de agua para miles de usos. Sin embargo, el agua para el consumo humano y uso doméstico debe estar libre de importantes concentraciones de contaminantes, haciéndose necesaria la implementación de algún tipo de sistema para su purificación (Sánchez, Cohim, \& Kalid).

El proceso de recolección de agua lluvia implica aprovecharla ya sea como apoyo o sustitución del sistema de agua potable, con el objetivo de evitar el desperdicio del vital líquido. En áreas donde el sistema de agua potable no existe, es irregular o no es lo suficientemente apta para el consumo humano, este sistema ha sido implementado con el fin de suministrar de alguna forma el servicio, siendo así un mecanismo que ofrece de alguna forma un uso sustentable del agua (Silva, Sousa, \& Carvalho 2015).

El agua posee características especiales en cuanto a las posibles formas de recolección superficial puesto que hay diversos tipos de contaminantes que pueden ser arrastrados por las aguas lluvias en forma de minerales naturales, sustancias orgánicas resultado de la presencia de animales o de las actividades antropogénicas. Los elementos que pueden presentarse en estas aguas naturales incluyen contaminantes microbianos como virus y bacterias, contaminantes inorgánicos como sales y metales, que pueden ser de origen natural, resultado de actividades humanas como el transporte o mala disposición de aguas residuales industriales o domésticas, de la producción petrolera y de gas, de la minería o de la agricultura, pesticidas y herbicidas, contaminantes radiactivos, y compuestos orgánicos volátiles y persistentes, entre otros (Gwenzi, Dunjana, Pisa, Tauro, \& Nyamadzawo; Thomas, Kirisits, Lye \& Kinney, 2014)

El proceso de reciclaje de agua lluvia implica los procesos de recolección, consistes en el uso de una superficie lo suficientemente apropiada como para la captación del agua lluvia, que por medio de pendientes dirigen al líquido hacia una canaleta hasta el área de almacenaje en un tanque. Esta área de recolección habrá de estar limpia, el mantenimiento que se le da a un techo promedio -por ejemplo-, bastará para asegurarse de que el agua que se obtiene carezca de una gran cantidad de micro-organismos dañinos para plantas, cultivos y animales, aunque no garantiza la potabilidad de la misma (Méndez et al., 2011). Limpieza de canaletas y bajadas de agua preexistentes llevarán el agua hasta el tanque de almacenamiento, con el objetivo de que partículas extrañas (basura, tierra, etc.) se queden en el fondo del mismo reduciendo al mínimo la contaminación por sólidos, previniendo tapones en el sistema del filtro diseñado, en las tuberías o sistemas de riego.

Estos diferentes usos, aparte del de consumo humano, han sido evaluados y marcados, como por ejemplo fuente de agua para servicio en edificios de grandes ciudades como Berlín, donde el agua lluvia es aprovechada en sistemas sanitarios y de riego mediante sistemas de recolección y almacenamiento diseñados para este 
propósito particular (Wisy, 2011). Un estudio realizado en el año 2007 en Nigeria (Rim-rukeh, Ikhifa, Okokoyo, \& Awatefe, 2007), evaluó el comportamiento de un filtro con partículas finas de hueso como material filtrante, donde se analizan los siguientes parámetros: $\mathrm{pH}$, temperatura, turbidez, SDT, SST, DBO, DQO y nitratos. Un análisis de pretratamiento de la corriente de alimentación mostró valores de $\mathrm{pH}(6,4)$, temperatura $\left(27,7^{\circ} \mathrm{C}\right)$; turbidez $(19,6 \mathrm{NTU}) ;$ SDT (906 mg/L); SST (28 mg/L); DBO (37 mg/L); DQO (41 mg/L) y nitratos $(21,17 \mathrm{mg} / \mathrm{L})$. La eficiencia de remoción del filtro fue evaluada para un período de cinco (5) días. El proceso de tratamiento alcanzó una eficiencia global 86,60\% de turbidez, SST $69,34 \%, 62,70 \%$ de TDS, $84,38 \%$ de DBO, DQO $41,88 \%$ y $51,20 \%$ de reducción de nitrato. De lo anterior se concluye que la adsorción de un filtro relleno con partículas finas de hueso, en términos de eficiencia de remoción de contaminantes en el agua Iluvia es alta (Rim-Rukeh, Ikhifa, Okokoyo, \& Awatefe, 2007). Sin embargo, todavía se encuentran lejos estas tecnologías de producir agua apta para consumo humano seguro (Lee, Yang, Han, \& Choi, 2010; Moon, Lee, Lee, Park, \& Jo, 2012).

\section{Materiales y métodos}

Para la recolección se usó una bajante la cual condujo el agua hacia un tanque de almacenamiento y cosecha. Esta fue objeto de determinación de parámetros físicos, químicos y biológicos, los cuales permitieron la caracterización del líquido. Para dichos análisis se tomó una muestra de dos litros de agua a la que se le determinaron diversos parámetros que se señalan a continuación: La determinación del $\mathrm{pH}$ se realizó de acuerdo con lo estipulado por el standard methods número 4500 , método electrométrico empleando un potenciómetro en laboratorio Schott Handylab, que permitió medir pH con una precisión de 0,01 unidades de pH. La turbiedad se midió según el SMWW 2130, usando un turbidimetro Merck Turbiquant, con una precisión de 0,0001 NTU. Sólidos suspendidos y disueltos se determinaron de acuerdo al SMWW 2540 C. La carga orgánica se estableció por medio de la demanda química de oxígeno (DQO) de acuerdo al método titulométrico de reflujo cerrado, SMWW 5220 C. Coliformes totales y fecales fueron determinadas con el método 9221B (Eaton, Franson, Association, Association, \& Federation, 2005).
A la cascarilla de arroz se le realizó un lavado y luego fue triturada. Para la caracterización en el laboratorio se realizaron pruebas de basicidad y acidez pesando por triplicado aproximadamente $0,5000 \mathrm{~g}$ del material adsorbente, colocando las cantidades antes pesadas en frascos con 50,0 ml de soluciones volumétricas valoradas $0,1 \mathrm{~N}$ de hidróxido de sodio, ácido clorhídrico, carbonato de sodio y bicarbonato de sodio por separado. Las soluciones se taparon y se dejaron a una temperatura de $25,0{ }^{\circ} \mathrm{C}$ durante 5 días con agitación manual ocasional. Concluido este tiempo, se tomaron muestras de $5,00 \mathrm{ml}$ de cada una de las soluciones y se titularon potenciométricamente por triplicado con solución estándar de ácido o base, según fuera el caso. Puesto que la concentración de grupos funcionales en el material adsorbente puede llegar a ser muy baja, es necesario que las soluciones a emplear tengan concentraciones menores a 0,05 N. Se realizó además la determinación de punto de carga cero (titulación de masas), pesando cantidades del material lignocelulósico en un rango de 0,1000 a 0,5000 g, cada una de las cuales fue colocada en frascos de $20,0 \mathrm{ml}$ y se les adicionó $10,0 \mathrm{ml}$ de cloruro de sodio 0,1 M. Las muestras fueron tapadas y se dejaron a una temperatura de $25,0^{\circ} \mathrm{C}$ con agitación constante durante 48 horas, tiempo después del cual se procedió a medir el pH de cada solución. Luego de la caracterización del material filtrante se fabricaron dos filtros; uno tuvo un soporte vegetal de luffa cylindrica comúnmente conocida como estropajo (filtro 1), que se esperaba evitara una rápida colmatación o compactación del filtro. El otro sistema solo se rellenó de material filtrante (filtro 2) en un espacio de $30 \mathrm{~cm}$ de los $40 \mathrm{~cm}$ que tuvo en total, teniendo además en los extremos espacios de retención para partículas de tamaño considerable y evitar su ingreso al filtro divididos en espacios de 5 $\mathrm{cm}$ en cada extremo, por medio de una tela de algodón que tiene la función adicional de repartir el flujo por toda la superficie del filtro como se muestra en la figura 1. Una vez montados los sistemas de filtración se realizó el seguimiento de los parámetros físicos y químicos mencionados por un período de 10 días y al final de este se hizo una evaluación microbiológica. 

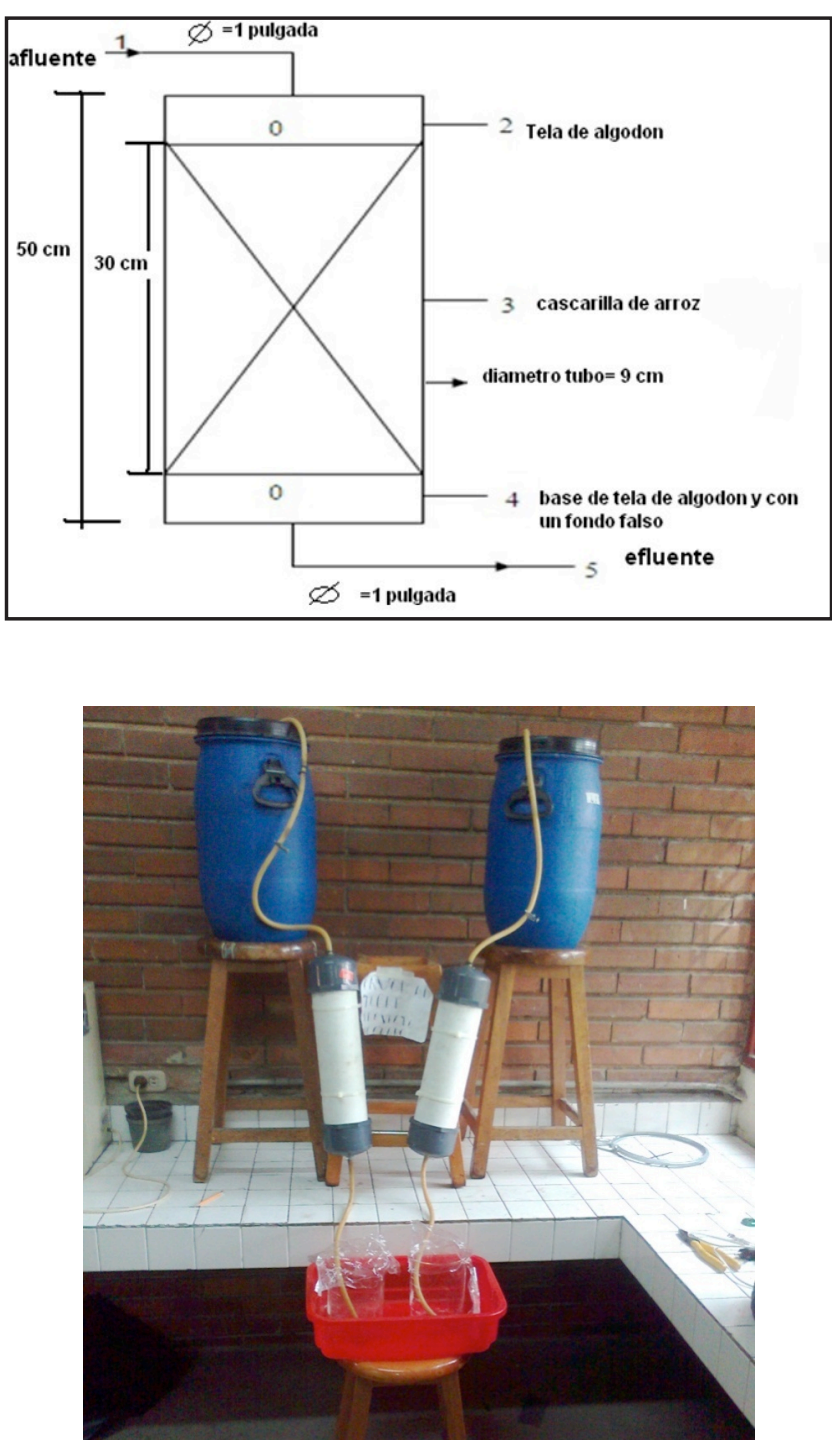

Figura 1. Esquema del sistema de filtr ación (arriba) y montaje experimental (abajo).

\section{Resultados}

\section{Caracterización inicial de la cascarilla de arroz}

La cascarilla muestra un punto de carga cero (pHPZC) de 4,82 , lo cual indica que la superficie del material posee sitios ácidos, los cuales podrían permitir procesos de adsorción de cationes que se encuentren en el agua lluvia. La acidez fue de 0,634 meq/g mientras que la basicidad fue de 0,183 meq/g, confirmando el carácter ácido de la superficie. La sumatoria de las dos al ser diferente de uno indica la existencia de superficie electrostáticamente neutra.

\section{Comportamiento de la calidad del agua en función del tiempo de operación}

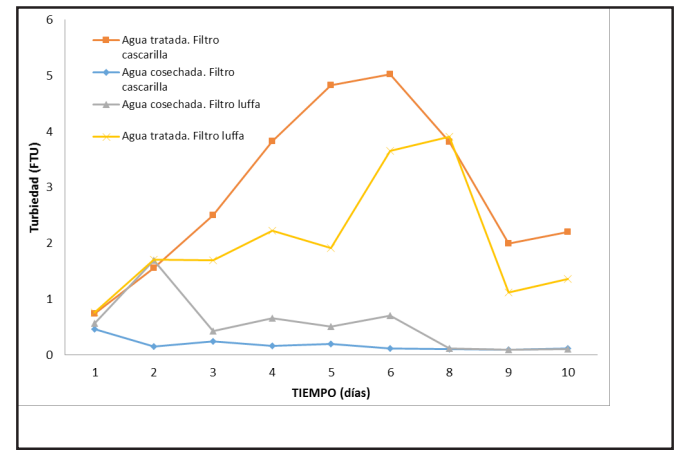

Figura 2. Turbiedad vs. tiempo

Al realizar la determinación de la turbiedad al agua cosechada antes de su paso por las columnas filtrantes, como se observa en la figura 2 , se evidenció que estas poseen niveles bajos con tendencia a reducir, pues debido a su almacenamiento se ha producido una sedimentación de sólidos presentes en el agua. En cambio al efectuar la valoración de la turbiedad a las aguas después de los filtros se hizo claro el aporte de elementos causantes de la misma por el material filtrante. En los últimos días de evaluación del filtro se observó una tendencia a la reducción de este parámetro a la salida del filtro, pero siempre obteniendo valores superiores al agua almacenada en los tanques de alimentación.

El comportamiento del pH para los dos filtros es diferente. Mientras el que no posee soporte de luffa cilíndrica tiende a bajar el $\mathrm{pH}$ hasta 0,4 unidades, el que tiene soporte tiene valores similares o incluso tiende a aportar basicidad sobre el final del tratamiento. Cabe considerar que la cascarilla al contar con sitios ácidos puede mediante procesos de adsorción remover las sustancias fuente de la alcalinidad del agua.

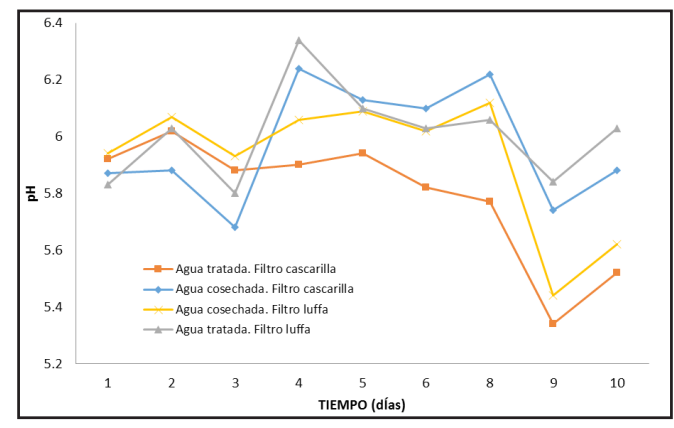

Figura 3. pH vs. tiempo 


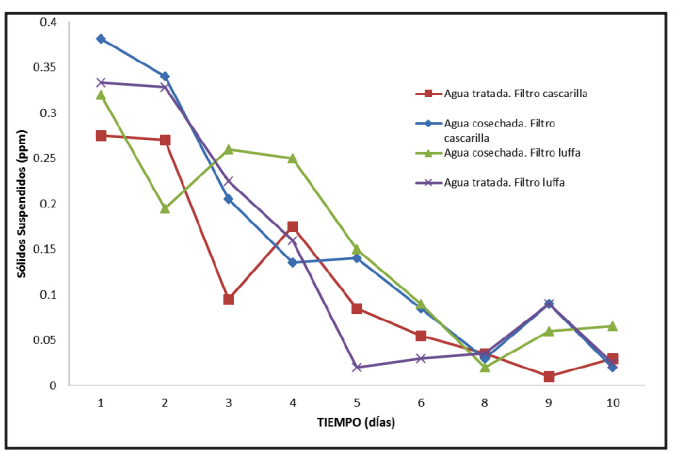

Figura 4. Sólidos suspendidos totales vs. tiempo

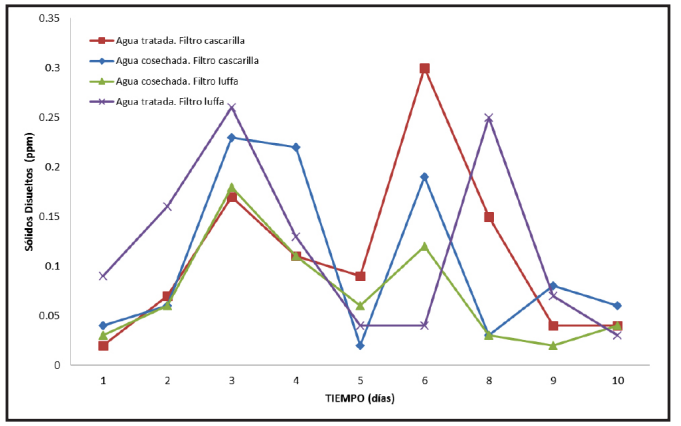

Figura 5. Sólidos disueltos totales vs. tiempo

En cuanto a sólidos suspendidos que se presentan en la figura 4, para el agua lluvia se obtuvieron valores iniciales de 0,32 ppm para el filtro 1 y 0,38 ppm para el filtro 2 con valores finales que están por debajo de 0,1 ppm. Existe una pequeña diferencia entre el agua tratada y sin tratar, sin embargo la remoción de este parámetro está determinada fundamentalmente por procesos de sedimentación dentro de los tanques de almacenamiento. Los sólidos disueltos de la figura 5 no son susceptibles a la remoción por procesos hidrodinámicos, sin embargo tampoco hay una remoción apreciable del mismo, puesto que es posible que los procesos de quimisorción no estén favorecidos por la carga superficial, generalmente negativa de los coloides que integran los sólidos disueltos.

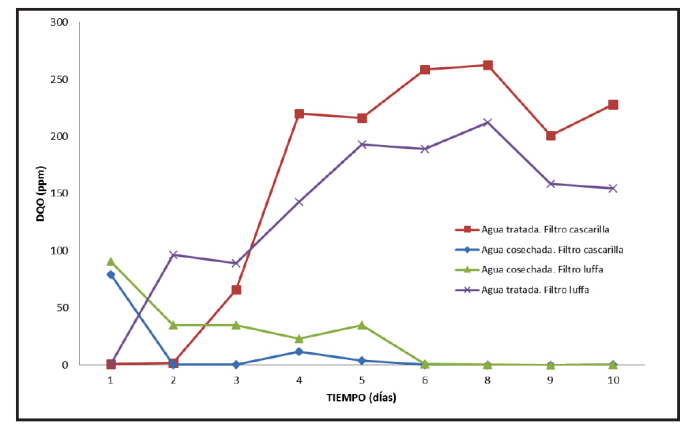

Figura 6. Demanda química de oxígeno vs. tiempo
El parámetro DQO, que permite establecer la carga orgánica presente en el agua, se ve negativamente afectado por el uso del filtro como se observa en la figura 6. Existe un aporte de materia orgánica el cual se ve más marcado en el filtro que tiene solo cascarilla que en el que posee estropajo como soporte. Esto puede deberse a que se presenta descomposición de la cascarilla, liberando materia orgánica, fenómeno que no es tan marcado en materiales como la luffa cilíndrica, la cual no presenta procesos de descomposición manifiestos en presencia de agua.

En cuanto a la presencia de microorganismos, en los resultados obtenidos al evaluar las unidades formadoras de colonias para coliformes totales y fecales para el último día de operación del filtro, no se obtuvo presencia de coliformes fecales, pero sí 41 unidades formadoras de colonia para coliformes totales, lo cual no la hace apta para consumo humano según la resolución 2115 del 2007.

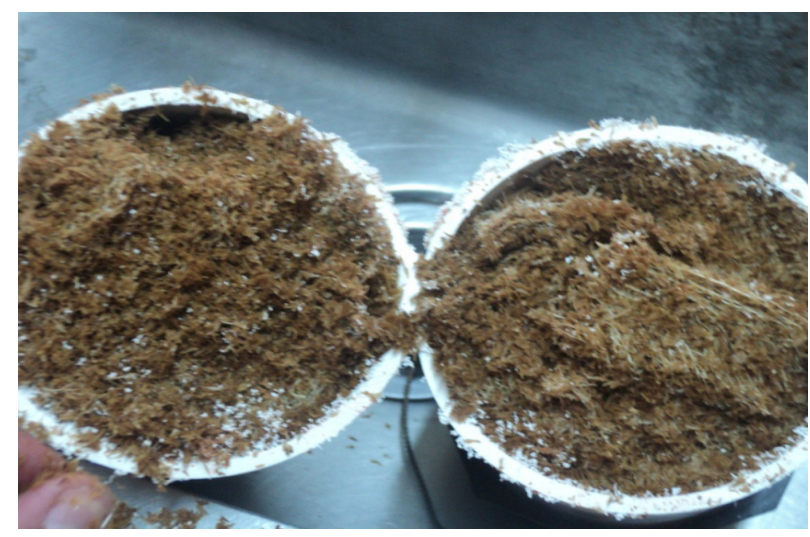

Figura 7. Material filtrante del filtro 1 después de 10 días

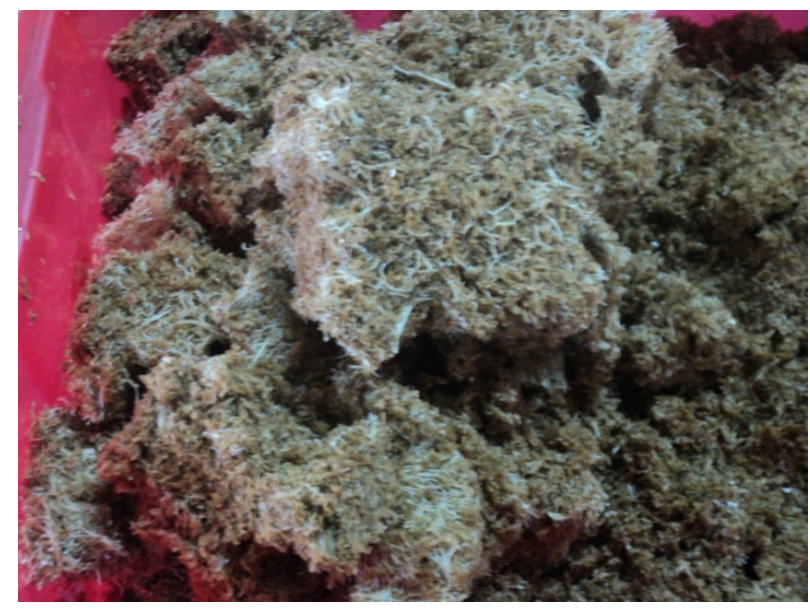

Figura 8. Material filtrante del filtro 2 después de 10 días 
El estado del material filtrante una vez pasado el período de evaluación reveló que hubo compactación del lecho, además de descomposición de la cascarilla al interior del filtro como se puede observar en las figuras 7 y 8 .
Finalmente, se realiza una comparación entre los parámetros del decreto 2115 de 2007 y algunos de los parámetros obtenidos en la evaluación de los sistemas de filtración, donde se puede apreciar que en su gran mayoría no cumplen con lo establecido por dicha resolución.

Tabla 1. Comparación de parámetros para agua de consumo y sistemas de filtración estudiados

\begin{tabular}{|l|c|c|c|}
\hline \multicolumn{1}{|c|}{ Parámetro } & Resultados filtros & $\begin{array}{c}\text { Resolución 2115 de } \\
\text { 2007. Valor máximo }\end{array}$ & Unidades \\
\hline Turbiedad & 3,5 & 2,0 & $\mathrm{FTU}$ \\
\hline $\mathrm{pH}$ & $5,4-6,2$ & $5,5-9,0$ & $\mathrm{UN}$ \\
\hline Nitratos & 0,8 & 10,0 & $\mathrm{mg} / \mathrm{l}$ \\
\hline Alcalinidad & 21,4 & 200,0 & $\mathrm{mg} / \mathrm{l} \mathrm{CaCO}$ \\
\hline Dureza & 0 & 300 & $\mathrm{mg} / \mathrm{CaCO}_{3}$ \\
\hline
\end{tabular}

\section{Conclusiones}

Al evaluar los resultados físicos, químicos y microbiológicos se encontró que los filtros no generaron una mejora en parámetros como pH, turbidez, SST y DQO. También se pudieron identificar de manera cualitativa cambios en el color del agua, la cual pasó de estar transparente a un color amarillento, y en el olor, donde se presentaron indicios de un proceso de descomposición que se pudo evidenciar en el aumento de carga orgánica en el efluente de los filtros. Debido a las condiciones que presentó el agua tratada por los filtros se evidencia que esta cumple con algunos de los niveles permisibles por la norma, lo cual nos indica que esta agua se puede emplear en actividades comunes como el riego de jardines, lavado de zonas duras o fachadas y emplearse en la descarga de sanitarios con una adecuada estructura hidráulica.

\section{Agradecimientos}

Los autores agradecen a la Universidad Libre y a la Universidad de Bogotá Jorge Tadeo Lozano por el soporte financiero para la realización de este trabajo.

\section{Referencias}

Ballén, J. A., Galarza, M. A., \& Ortiz, R. O. (2006). Historia de los sistemas de aprovechamiento de agua Iluvia en Colombia. Paper presented at the Seminario Iberoamericano sobre sistemas de abastecimiento urbano de agua.
Eaton, A. D., Franson, M. A. H., Association, A. P. H., Association, A. W. W., \& Federation, W. E. (2005). Standard Methods for the Examination of Water \& Wastewater: American Public Health Association.

Guozhen, Z., Yuanchao, Y., Xiaodong, L. I. U., \& Weina, Z. (2011). Research and Application of harvested rainwater in the villages and towns of China Loess Plateau region. Energy Procedia, 5(0), 307-313. doi: http://dx.doi.org/10.1016/j.egypro.2011.03.053

Gwenzi, W., Dunjana, N., Pisa, C., Tauro, T., \& Nyamadzawo, G. (2015). Water quality and public health risks associated with roof rainwater harvesting systems for potable supply: Review and perspectives. Sustainability of Water Quality and Ecology. doi: http://dx.doi.org/10.1016/j.swaqe.2015.01.006

Lee, J. Y., Yang, J.-S., Han, M., \& Choi, J. (2010). Comparison of the microbiological and chemical characterization of harvested rainwater and reservoir water as alternative water resources. Science of The Total Environment, 408(4), 896-905. doi: http://dx.doi.org/10.1016/j.scitotenv.2009.11.001

Méndez, C. B., Klenzendorf, J. B., Afshar, B. R., Simmons, M. T., Barrett, M. E., Kinney, K. A., \& Kirisits, M. J. (2011). The effect of roofing material on the quality of harvested rainwater. Water Research, 45(5), 2049-2059. doi: http://dx.doi.org/10.1016/j. watres.2010.12.015 
Moon, S.-H., Lee, J.-Y., Lee, B.-J., Park, K.-H., \& Jo, Y.-J. (2012). Quality of harvested rainwater in artificial recharge site on Jeju volcanic island, Korea. Journal of Hydrology, 414-415(0), 268-277. doi: http:// dx.doi.org/10.1016/j.jhydrol.2011.10.041

Rim-Rukeh, A., Ikhifa, G. O., Okokoyo, A. P., \& Awatefe, J. K. (2007). Treatment of harvested rainwater in a pilot scale fixed-bed filled with bone char. Journal of Applied Sciences Research, 3(8), 690-694.

Sánchez, A. S., Cohim, E., \& Kalid, R. A. (2015). A review on physicochemical and microbiological contamination of roof-harvested rainwater in urban areas. Sustainability of Water Quality and Ecology. doi: http://dx.doi.org/10.1016/j.swaqe.2015.04.002
Silva, C. M., Sousa, V., \& Carvalho, N. V. (2015). Evaluation of rainwater harvesting in Portugal: Application to single-family residences. Resources, Conservation and Recycling, 94(0), 21-34. doi: http:// dx.doi.org/10.1016/j.resconrec.2014.11.004

Thomas, R. B., Kirisits, M. J., Lye, D. J., \& Kinney, K. A. (2014). Rainwater harvesting in the United States: a survey of common system practices. Journal of Cleaner Production, 75(0), 166-173. doi: http://dx. doi.org/10.1016/j.jclepro.2014.03.073

Wisy, A. G. (2011). Rainwater harvesting: Harnessing the elements. Filtration + Separation, 48(5), 41-43. doi: http://dx.doi.org/10.1016/s0015-1882(11)70213-0 